\title{
Suprasternal notch needle decompression to treat severe pneumomediastinum
}

A 56-year-old man underwent endoscopic submucosal tunnel dissection (ESTD) for an esophageal submucosal tumor. Because the patient had the co-morbidity of moderate chronic obstructive pulmonary disease (COPD), room-air insufflation instead of carbon dioxide insufflation was used during the operation. We began the procedure by making a longitudinal incision $4 \mathrm{~cm}$ above the targeted tumor; a submucosal tunnel was then created downward to the distal end of the tumor. The tumor was dissected en bloc from the surrounding submucosal tissues and smooth muscle fibers ( $\bullet$ Fig. 1 a), and retrieved orally. The initial mucosal incision was closed by clips.

During tumor dissection, there was iatrogenic injury to the muscularis propria ( $\bullet$ Fig.1b) and mediastinal emphysema occurred immediately as the esophagus has no serosa. Severe subcutaneous emphysema of eyelid, face, neck, and submandibular region was observed; palpation of the chest and neck was characteristic and gave the impression of palpating "snow." The patient's heart rate soared to 130 beats/min while his blood pressure dropped to $80 / 60 \mathrm{mmHg}$ and oxygen saturation down to $80 \%$.

We attempted needle decompression before the patient would have been transferred to surgery. After topical disinfection, the needle of a 22-gauge syringe that was preloaded with $2 \mathrm{ml}$ of normal saline was inserted into the skin over the superior border of the sternal bone (the jugular notch). The needle was advanced until air could be aspirated into the syringe (bubbles in the normal saline). The loading device was then removed and air continued to be expelled from the chest ( Fig. 2 , - Video 1). Meanwhile, the subcutaneous emphysema was significantly relieved. The needle was removed when there was no further escape of air. Antibiotics were given for 2 days postoperatively, and the patient was discharged uneventfully after 5 days.

\section{Video 1}

Needle decompression at the suprasternal notch for treatment of severe pneumomediastinum.

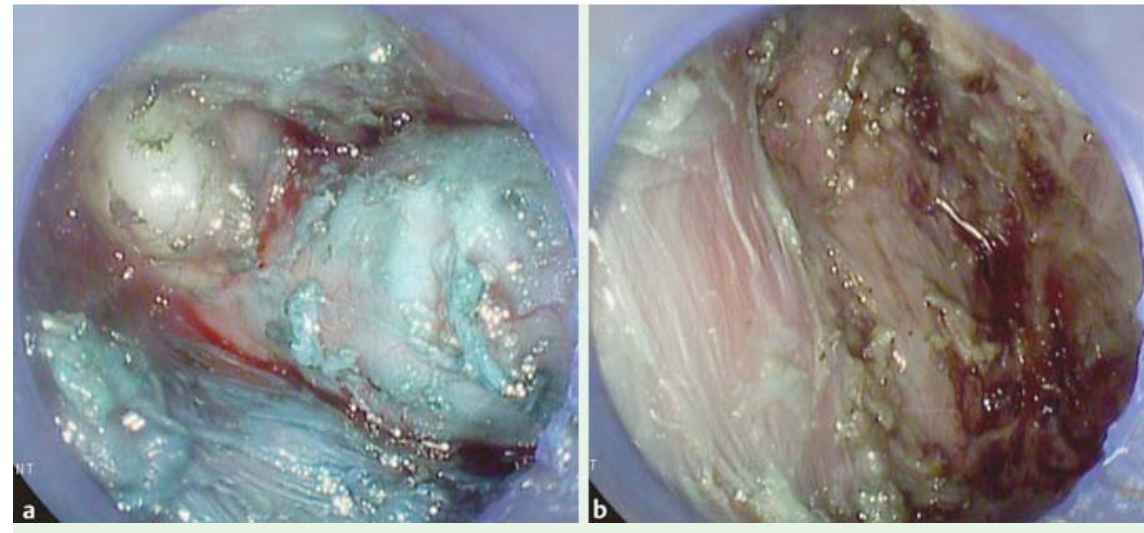

Fig. 1 a The esophageal submucosal tumor seen in the submucosal tunnel during endoscopic submucosal tumor dissection (ESTD). b The iatrogenic injury to the muscularis propria layer after tumor resection.

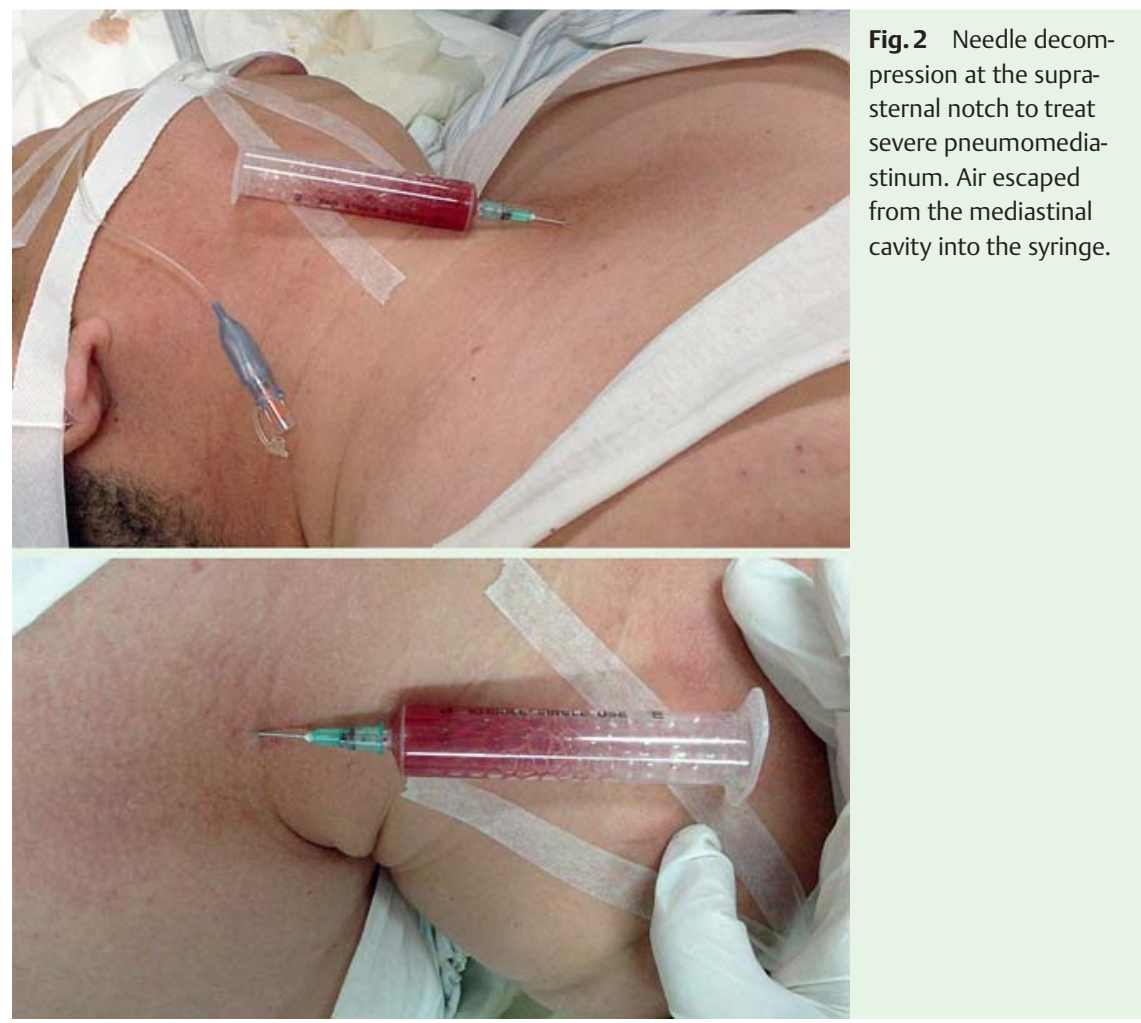

The emergence of the endoscopic resection of esophageal lesions has occasioned a new iatrogenic cause of mediastinal emphysema. In severe cases complicated by respiratory and circulatory failure, needle/tube aspiration at the intercostal space is less effective and surgical intervention may be required. In this case, we found the "Achilles heel" of severe med- iastinal emphysema - the suprasternal notch - and released the trapped air quickly and effectively.

Endoscopy_UCTN_Code_CPL_1AH_2AH

Competing interests: None 


\section{Jiaoyang Lu1,2, *, Minhua Zheng1, *,} Xiuli Zuo ${ }^{2}$, Yanqing $\mathbf{L i}^{2}$, Xuefeng $\mathbf{L u}^{2}$

${ }^{1}$ Department of General Surgery, Ruijin Hospital, Shanghai Jiao Tong University School of Medicine, Shanghai, China

${ }^{2}$ Department of Gastroenterology, Qilu Hospital, Shandong University School of Medicine, Shandong, China

\footnotetext{
* Jiaoyang Lu and Minhua Zheng contributed equally to this manuscript.
}

Bibliography

Dol http://dx.doi.org/

10.1055/s-0034-1377441

Endoscopy 2014; 46: E343-E344

(C) Georg Thieme Verlag KG

Stuttgart · New York

ISSN 0013-726X
Corresponding author

\section{Xuefeng Lu, MD}

Department of Gastroenterology, Qilu Hospital Shandong University School of Medicine Jinan, Shandong

China, 250012

Fax: +86-531-82166095

lu0801shanyi@163.com 